\title{
Shared Neural Mechanisms for the Evaluation of Intense Sensory Stimulation and Economic Reward, Dependent on Stimulation-Seeking Behavior
}

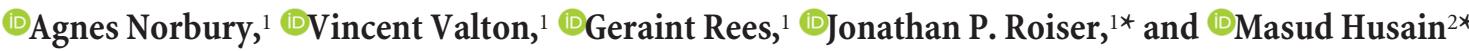 \\ ${ }^{1}$ Institute of Cognitive Neuroscience, University College London, London WC1N 3AR, United Kingdom, and ${ }^{2}$ Department of Experimental Psychology and \\ Nuffield Department of Clinical Neurosciences, University of Oxford, Oxford OX1 3UD, United Kingdom
}

\begin{abstract}
Why are some people strongly motivated by intense sensory experiences? Here we investigated how people encode the value of an intense sensory experience compared with economic reward, and how this varies according to stimulation-seeking preference. Specifically, we used a novel behavioral task in combination with computational modeling to derive the value individuals assigned to the opportunity to experience an intense tactile stimulus (mild electric shock). We then examined functional imaging data recorded during task performance to see how the opportunity to experience the sensory stimulus was encoded in stimulation-seekers versus stimulation-avoiders. We found that for individuals who positively sought out this kind of sensory stimulation, there was common encoding of anticipated economic and sensory rewards in the ventromedial prefrontal cortex. Conversely, there was robust encoding of the modeled probability of receiving such stimulation in the insula only in stimulation-avoidant individuals. Finally, we found preliminary evidence that sensory prediction error signals may be positively signed for stimulation-seekers, but negatively signed for stimulation-avoiders, in the posterior cingulate cortex. These findings may help explain why high intensity sensory experiences are appetitive for some individuals, but not for others, and may have relevance for the increased vulnerability for some psychopathologies, but perhaps increased resilience for others, in high sensation-seeking individuals.
\end{abstract}

Key words: addiction; decision-making; impulsivity; individual differences; sensation-seeking; value

Significance Statement

People vary in their preference for intense sensory experiences. Here, we investigated how different individuals evaluate the prospect of an unusual sensory experience (electric shock), compared with the opportunity to gain a more traditional reward (money). We found that in a subset of individuals who sought out such unusual sensory stimulation, anticipation of the sensory outcome was encoded in the same way as that of monetary gain, in the ventromedial prefrontal cortex. Further understanding of stimulation-seeking behavior may shed light on the etiology of psychopathologies such as addiction, for which high or low sensation-seeking personality has been identified as a risk factor.

\section{Introduction}

Why do some people place a high value on unusual sensory experiences, such as riding rollercoasters, fast driving, or consum-

\footnotetext{
Received March 29, 2016; revised July 18, 2016; accepted July 19, 2016

Author contributions: A.N., G.R., J.P.R., and M.H. designed research; A.N. performed research; A.N. and V.V. analyzed data; A.N., V.V., G.R., J.P.R., and M.H. wrote the paper.

*J.P.R. and M.H. are co-senior authors.

This work was supported by the Wellcome Trust and the United Kingdom Medical Research Council.

The authors declare no competing financial interests.

This article is freely available online through the J Neurosci Author Open Choice option.

Correspondence should be addressed to Agnes Norbury at the above address. E-mail: aen31@cam.ac.uk.

A. Norbury's present address: Computational and Biological Learning Laboratory, Department of Engineering, University of Cambridge, Trumpington Street, Cambridge CB2 1PZ, UK.

DOI:10.1523/JNEUROSCI.1048-16.2016

Copyright $\odot 2016$ Norbury et al.

This is an Open Access article distributed under the terms of the Creative Commons Attribution License Creative Commons Attribution 4.0 International, which permits unrestricted use, distribution and reproduction in any medium provided that the original work is properly attributed.
}

ing mind-altering substances? The trait of sensation-seeking refers to stable individual differences in preference for "intense and unusual sensory experiences" (Zuckerman, 1994). Both high and low levels of sensation-seeking personality have been identified as risk factors for psychopathologies with high social cost (Roberti, 2004; Norbury and Husain, 2015), yet the neural mechanisms underlying how individuals assign value to intense sensory experiences are unclear.

In this study, we used a novel behavioral task in conjunction with computational modeling and functional magnetic resonance imaging ( $\mathrm{fMRI}$ ) to probe individual differences in regional brain activity during performance of stimulation-seeking and stimulation-avoiding behavior. This approach allowed us to address directly for the first time in humans whether the opportunity to experience intense sensory stimulation activates brain regions typically considered part of "reward" circuitry (Haber 
A

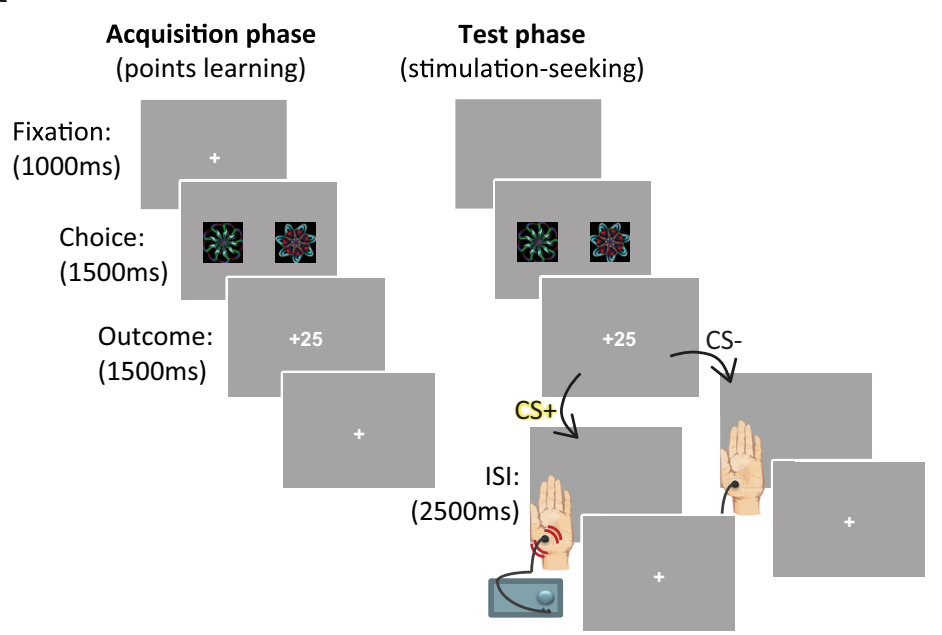

C

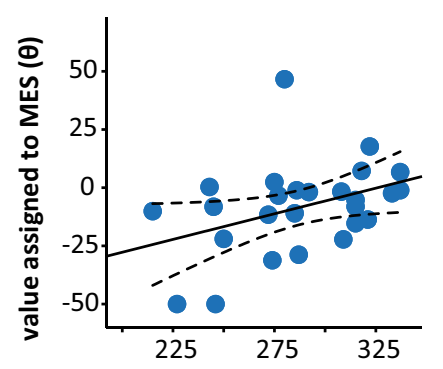

self-reported sensation-seeking

F

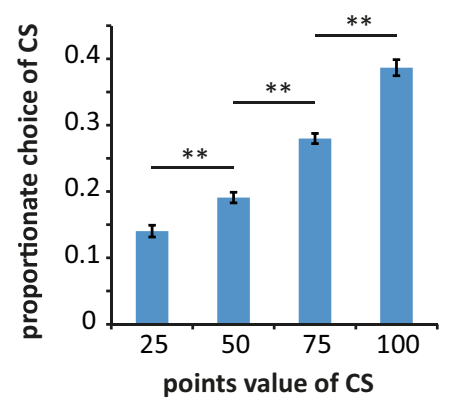

B

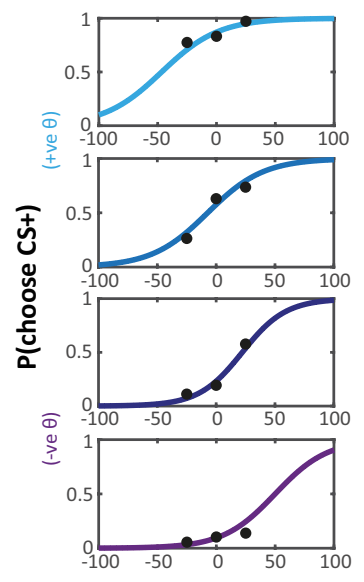

relative points value $\mathrm{CS}+$

E

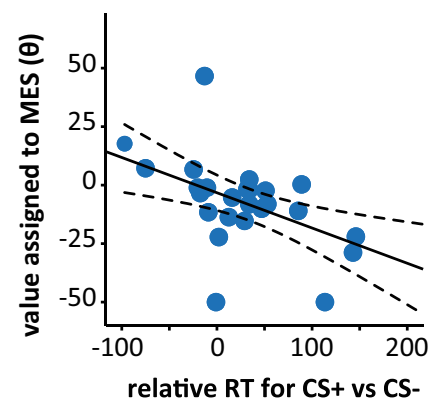

G

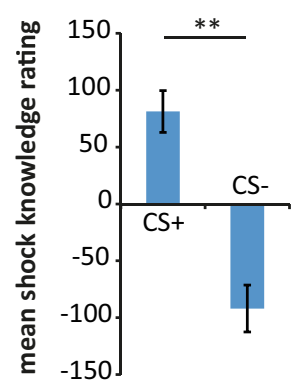

H

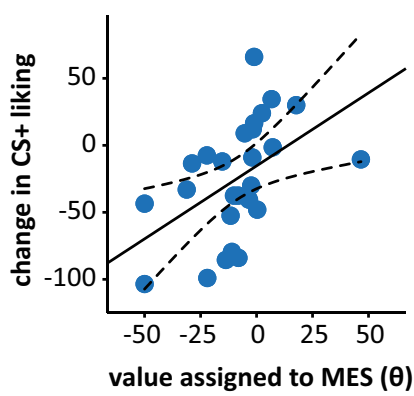

Figure 1. Stimulation-seeking task and summary of prescan and in-scanner behavior. $A$, Stimulation-seeking task. Participants first learned the points values associated with different abstract choice stimuli or CSs (acquisition phase). Half the choice stimuli then became associated with the chance of receiving MES to the hand (became $C S+s, P(M E S \mid C S+)=0.75)$, while the other half had no additional sensory consequences (became $C S-S, P(M E S \mid C S-)=0$ ). Observing how the opportunity to receive this additional intense tactile stimulation affected participants' choice during these test-phase trials thus allowed derivation of the precise value individuals assigned to the opportunity to receive the extrasensory stimulation ( $\theta$ ). $B$, Individual choice functions fit to test-phase data from four representative participants, ranging from strongly stimulation-seeking (large positive $\theta$ value; top) to strongly stimulationavoiding (large negative $\theta$ value; bottom). A leftward shift in the curve denotes assignment of positive additional value to the opportunity to experience the MES, and a rightward shift a negative value, with the gradient reflecting an individual's choice stochasticity parameter, $\beta$. $C$, Relationship between value assigned to opportunity to experience the MES and self-reported sensation-seeking score (SSS-V-R total score; $r=0.391, p=0.048$ ). $\boldsymbol{D}$, Relationship between MES value (i.e., $\theta$ ) and relative choice reaction time for MES-associated

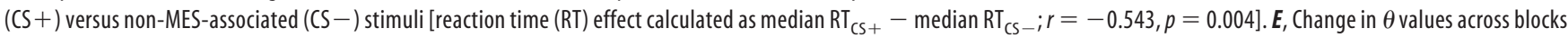
inside the scanner, illustrated separately for stimulation-seeking ( $S S$; overall $\theta>0, N=8)$ and stimulation-avoiding $(S A$; overall $\theta<0, N=18)$ individuals. $F$, Proportionate choice of CSs according to their points value, averaged across all acquisition-phase (points learning) trials. In this initial phase (completed before entering the scanner), all CSs were pitted against each other repeatedly in a tournament design. On each trial, participants chose between two CSS, and were then awarded the points value of their chosen CS. Overall proportionate choice of each CS is therefore taken as an index of learning about their points (economic) value. G, Mean "shock knowledge" ratings (ratings on a VAS ranging from "no chance of shock" at -300 to "chance of shock" at +300) for CS + (MES-associated) and CS - (non-MES-associated) stimuli (ratings were completed following completion of initial shock-learning trials, before entering the scanner). $\boldsymbol{H}$, Relationship between mean change in VAS "liking" rating of CS + $s$ following introduction of the MES (before entering the scanner), and MES value $(\theta)$ calculated from all scanner trials. Error bars represent SEM; dotted lines represent $95 \%$ confidence intervals; $N=26 .{ }^{* *} p<0.001$. 
and Knutson, 2010) in individuals for whom such stimulation appears to be appetitive. Specifically, we tested the hypothesis that intense sensory stimulation (sensory reward) and higher economic value (monetary reward) would be encoded in the same neural circuitry in individuals who sought out such stimulation-in this case, mild electric shock (MES).

In a paradigm designed to be analogous to an operant stimulation-seeking task from the animal literature (Olsen and Winder, 2009), participants first learned the points (economic) values associated with different abstract fractal images. They then learned that choosing some of these images could result in the receipt of nonpainful electrical stimulation to the hand (Fig. 1A). Individuals' preference for each fractal or abstract choice stimulus was then probed during collection of fMRI data. A simple computational model based on participants' choice data and the relative monetary value of choice options allowed us to derive the additional economic value-positive or negative- that they assigned to the opportunity to receive the intense tactile stimulation (MES). We were therefore able to monitor during task performance how regional changes in blood oxygenation level-dependent (BOLD) signals varied with this quantitative behavioral index of sensation-seeking.

We hypothesized that activity in the ventral striatum (vS) and ventromedial prefrontal cortex (vmPFC) may encode responses to both MES-associated stimuli and economic reward, selectively in stimulation-seekers. Such common value coding might explain why intense sensory experiences are powerful positive motivators for high sensation-seeking individuals. When economic value for other types of choice has been investigated in the past, BOLD signal in the vmPFC and vS has been identified as representing the expected value of options (Knutson et al., 2001; Abler et al., 2006; Levy and Glimcher, 2012; Clithero and Rangel, 2014) and even valued memories of past experiences (Speer et al., 2014). Although the interpretation of these results has been questioned (Hayden and Heilbronner, 2014; O'Doherty, 2014), the vmPFC in particular has been identified in analyses probing common representation across numerous different kinds of rewards (Levy and Glimcher, 2012; Clithero and Rangel, 2014), indicating that this region may be involved in computations using a common value currency.

Conversely, we hypothesized that stimulation-avoiders may experience (anticipation of) the MES as more aversive and/or salient. Thus, we predicted that a brain region that has consistently been implicated in responding to aversive stimuli, the insula (Büchel et al., 1998; Nitschke et al., 2006; Menon and Uddin, 2010), would exhibit increased activity when choosing MESassociated options in these individuals. This might help explain why anticipation of intense sensory stimulation evokes anxiety and avoidance-like responses in low sensation-seekers, but not high-sensation seekers (Lissek and Powers, 2003; De Pascalis et al., 2007; Norbury et al., 2015).

\section{Materials and Methods \\ Participants}

Twenty-seven right-handed healthy volunteers (18 female; mean age, 22.6 years; SD, 2.9) were recruited via Internet advertisement. Exclusion criteria consisted of any current or past neurological or psychiatric illness, or head injury. All participants provided written informed consent and the study was approved by the University College London ethics committee. Data from one participant had to be discarded due to technical difficulties on the scan day, yielding a final $N$ of 26 .

\section{Table 1. Details of trial types for the stimulation-seeking task (test phase)}

\begin{tabular}{lll}
\hline Trial type & Stimuli & Points values \\
\hline 1 & CS1 (+) vs CS2 (-) & $25(+)$ vs $50(-)$ \\
2 & CS2 (-) vs CS3 (+) & $50(-)$ vs $75(+)$ \\
3 & CS3 (+) vs CS4 (-) & $75(+)$ vs $100(-)$ \\
4 & CS5 (-) vs CS6 (+) & $25(-)$ vs $50(+)$ \\
5 & CS6 (+) vs CS7 (-) & $50(+)$ vs $75(-)$ \\
6 & CS7 (-) vs CS8 (+) & $75(-)$ vs $100(+)$ \\
7 & CS1 (+) vs CS5 (-) & $25(+)$ vs $25(-)$ \\
8 & CS2 (-) vs CS6 (+) & $50(-)$ vs $50(+)$ \\
9 & CS3 (+) vs CS7 (-) & $75(+)$ vs $75(-)$ \\
10 & CS4 (-) vs CS8 (+) & $100(-)$ vs $100(+)$ \\
\hline
\end{tabular}

CS, conditioned stimulus; $(+)$, stimulus associated with chance of receiving the MES $(C S+) ;(-)$, no chance of receiving the MES (CS-).

\section{Stimulation-seeking task}

The stimulation-seeking task was administered as described in Norbury et al. (2015), summarized in Figure $1 A$ and Table 1. The task is designed to probe the precise economic value (positive or negative) that participants assign to the opportunity to receive an "intense" sensory stimulus [mild, nonpainful electric shock (MES)]. Participants were instructed that they would be paid a cash bonus at the end of the task, depending on how many points they managed to accrue. Thus points had real-world monetary (economic) value.

Before completing the task, participants completed a "work-up" procedure designed to ensure that the MES was approximately similar in subjective intensity across participants. Specifically, participants received a series of single stimulation pulses, starting at a very low amplitude $(0.5 \mathrm{~mA}$; generally reported by participants as being only just detectable) and gradually increasing in current strength until the stimulation was rated as 6 of 10 on a visual analog scale (VAS) ranging from 0 ("just detectable") to 10 ("painful or unpleasant"). At this level, all participants endorsed a description of the sensation as being "stimulating but not painful." This procedure was repeated twice for each participant to ensure consistency.

\section{Design}

During a prescreening session, participants completed a baseline run of the stimulation-seeking task and were screened for functional imaging contraindications. Volunteers then completed a revised form of the Sensation-Seeking Scale version V (Zuckerman, 1994; Gray and Wilson, 2007) and the trait scale from the State-Trait Anxiety Inventory (STAI; Spielberger et al., 1970). Information about cigarette and alcohol consumption, recreational drug use, and frequency of engagement in gambling-related activities was also collected. Where appropriate (alcohol or recreational drug use, $>0$ ), participants then completed the Alcohol Use Disorder Identification and Drug Abuse Screening Tests (DAST; Skinner, 1982; Saunders et al., 1993). Finally, a standardized measure of nonverbal IQ was administered (Raven's 12-item Advanced Progressive Matrices; Pearson Education, 2010). Additional exclusion criteria at this stage consisted of unsuitability for functional imaging, STAI trait score indicative of a current anxiety disorder, DAST score indicative of a past or present substance-use disorder, or self-reported recreational drug use within the last month.

On the scan day, participants completed a version of the stimulationseeking task used in Norbury et al. (2015), modified to minimize the amount of learning occurring during the scanning stage. During a period of prescan training, participants first learned the points values associated with each fractal image (conditioned stimulus, or CS), using a tournament design similar to that used by Lawson et al. (2014). On each trial, participants chose between a pair of CSs (with left/right position randomly assigned), and were then awarded the number of points associated with their chosen CS (Fig. 1A). Each of eight CSs was pitted against each other CS four times, yielding 112 trials in total. Overall proportionate choice of each CS, according to its points value, was then taken as an index of learning. Participants were also exposed to the CS-MES contingencies before entering the scanner. Specifically, they completed 10 of each "equal points" trial type (Table 1, trial types 7-10), where one of 
each choice pair was associated with a chance of receiving the MES $(P=$ 0.75 , the CS +$)$, and the other was associated with no chance of receiving the $\operatorname{MES}(P=0$, the CS $-; 40$ trials in total).

Participants further completed VAS "liking" ratings of each CS at three stages: (1) before starting the task, (2) after learning the CS-points value associations, and (3) after exposure to the CS-MES contingencies (on a scale ranging from "like" to "dislike"). They also completed "shock knowledge" ratings of each CS at the end of the prescan block, on a VAS ranging from "chance of shock" to "no chance of shock." Finally, participants additionally rated how they felt about the MES itself on a VAS ranging from "like" to "dislike."

Participants then proceeded to the in-scanner phase. First, all volunteers repeated the shock amplitude work-up procedure (see above) to attempt to match subjective intensity of the mild electric stimulation across contexts and apparatus setups. Once inside the scanner, participants completed three blocks of 100 test phase trials (Fig. $1 A$, all trial types). For each 100-trial block, they completed 13 of each type of equal points trial ( 52 trials total) and eight of each remaining trial type ( 48 trials total).

\section{Apparatus}

Apparatus for the prescreening and prescanner testing was as described in our previous study (Norbury et al., 2015). Specifically, electrical stimulation was generated using a Digitimer DS7A constant current stimulator (Digitimer), with output triggered remotely from a desktop computer via parallel port using Matlab. Pulse duration was $2000 \mu$ s and stimulation was delivered to participants via a pair of disposable $\mathrm{Ag} / \mathrm{AgCl}$ EEG-EMG electrodes with $15 \times 20 \mathrm{~mm}$ self-adhesive pads (Spes Medica) attached $\sim 1 \mathrm{~cm}$ apart on the thenar eminence of the nondominant hand.

For the functional imaging stage, participants wore disposable, radiotranslucent, pregelled electrodes ( $\mathrm{Ag} / \mathrm{AgCl}$ laminated, carbon composition contact; Biopac Systems) on the thenar eminence of their left hand. Electrodes were attached to a Digitimer DS7A constant current stimulator (as before) via a radiotranslucent carbon fiber clip lead (Biopac). Stimulator output was controlled via optic fiber projection from a stimulus PC in the scanner control room. Visual stimuli were displayed via back projection on to a head-set mirror worn by participants inside the scanner. Visual stimulus presentation and MES delivery were controlled via Cogent 2000 v1.30, run in Matlab.

\section{Behavioral analysis}

Behavioral data were analyzed using the model described in Norbury et al. (2015). Specifically, it was assumed that the choice between the CS+ and CS - could be represented as follows (Eq. 1):

$$
\begin{gathered}
\mathrm{V}_{\mathrm{CS}+}=\mathrm{R}_{\mathrm{CS}+}+\theta \\
\mathrm{V}_{\mathrm{CS}-}=\mathrm{R}_{\mathrm{CS}-}
\end{gathered}
$$

where $R_{\mathrm{X}}$ is the points value of stimulus $X, \theta$ is the additional value (in points) assigned to the opportunity to receive the MES (positive or negative), and $V_{X}$ represents the overall value of each option. This model was then fitted across all test phase choice data for each participant via a sigmoidal choice (softmax) function as follows (Eq. 2):

$$
P(\text { choose CS }+)=1 /\left(1+\exp \left(-\beta *\left(V_{\mathrm{CS}^{+}}-V_{C^{-}}\right)\right)\right) .
$$

Values of the free parameters $\theta$ and $\beta$ (the softmax temperature parameter, a measure of choice stochasticity) were fitted to the data on a subject-by-subject basis using maximum likelihood estimation (MLE).

For further analysis of behavior inside the scanner, a trial-by-trial model of task behavior was implemented, in order to regress model components against trial-by-trial fluctuations in BOLD signal (Daw, 2011). Choice data from the prescan "test" phase (first exposure to CSMES pairings) was also included in the model to fully account for experience of CS-MES contingencies at the start of the first scanner trial [data from the prescan "acquisition" (points learning) phase were not included; therefore, it was assumed that points values of CSs were fully learned by this stage].

For the modeled data (prescan test phase and all in-scanner trials), it was assumed that the value of each $\mathrm{CS}\left(V_{\mathrm{CS}}\right)$ on each trial $(t)$ could be represented as follows (Eq. 3): $V_{C S, t}=R_{C S}+S A V_{C S, t} * \theta$, where $R_{C S}$ represents the points value of each CS, $\theta$ again represents the additional value (positive or negative) participants assign to opportunity to receive the MES, and $S A V_{C S}$ represents modeled internal probability of receiving a shock, given choice of that CS (shock associative value, SAV, of each CS) on each trial.

Following the outcome of trial $t$, the SAV of each CS $\left(S A V_{C S}\right)$ was updated according to the actual trial outcome (shock vs no shock received) and a simple Rescorla-Wagner learning rule as follows (Eq. 4): $S A V_{C S, t+1}=S A V_{C S, t}+\alpha\left(S-S A V_{C S, t}\right)$.

Simply, after the outcome of each trial $t$, the SAV of the chosen CS is updated to a new value, which is equal to the sum of the previous SAV for that $C S\left(S A V_{C S, t}\right)$, plus a prediction error term multiplied by a learning rate $(\alpha)$. The prediction error term represents the difference between the expected sensory outcome (previous probability estimate for receipt of the MES, given choice of that CS, i.e., $\left.S A V_{C S, t}\right)$ and the actual sensory outcome ( $S$; with a value of 1 for shock or 0 for no shock received). This model was then fit across choice data from each participant via the sigmoidal link (softmax) function described in Equation 2.

Values of the free parameters $(\theta, \alpha$, and the softmax parameter $\beta)$ were then estimated for each participant using MLE. To decrease the likelihood of outliers, we implemented an additional stage of maximum a posteriori (MAP) likelihood estimation on parameter estimates (Daw, 2011). MAP represents a hierarchical Bayesian approach to parameter estimation, such that the group of parameter estimates derived at the first stage (MLE step) are subsequently used to estimate the true population distribution of parameter values (in this case, assumed to be Gaussian). This distribution then becomes the prior likely distribution of parameters for use in a second round of inference using MAP.

Computational modeling of choice data was implemented in Matlab r2015a (Mathworks) and used a nonlinear function optimization solver to infer the optimal parameters for each individual (Matlab function fmincon). Other statistical analyses were performed in SPSS Statistics 19.0 (IBM). Associations of model parameters with non-normally distributed self-report data were tested using nonparametric statistical tests. Where appropriate, $p$ and $\mathrm{df}$ were adjusted for violation of Levene's test for equality of variances using the Welch-Satterthwaite method. All reported analyses are two-tailed, with an $\alpha$ of 0.05 .

\section{MRI data acquisition and analysis}

\section{Acquisition parameters}

Functional imaging data were collected on a 3T Magnetom TIM Trio scanner (Siemens Healthcare) equipped with a 32-channel head coil. To correct for inhomogeneities in the static magnetic field, field maps were acquired using a double-echo FLASH (gradient echo) sequence (short $\mathrm{TE}=10 \mathrm{~ms}$, long $\mathrm{TE}=12.46 \mathrm{~ms}, 64 \times 2 \mathrm{~mm}$ slices, $3 \times 3 \mathrm{~mm}^{2}$ resolution). Three functional scanning sessions, consisting of six dummy volumes and 188 functional volumes, were then acquired using a gradient echo-planar imaging (EPI) sequence optimized for signal in the prefrontal and orbitofrontal cortex (voxel size, $3 \times 3 \times 3 \mathrm{~mm}$; volume repetition time, $3.36 \mathrm{~s}$; echo time, $30 \mathrm{~ms}$; matrix size, $64 \times 74$; tilt, $-30^{\circ}$; $Z$-shim, 1.4; slices per volume, 48 ; whole-brain coverage; Weiskopf et al., 2006). A T1-weighted modified driven equilibrium Fourier transform (MDEFT) structural scan was also acquired (Deichmann et al., 2004).

\section{Preprocessing}

MRI data were analyzed using SPM8 (Wellcome Trust Centre for Neuroimaging, London, UK), run in Matlab. The first six (dummy) volumes of each functional session were discarded to allow for T1 equilibration. Functional images were intensity-bias corrected (intensity profiles were flattened across images using the bias correction procedure implemented by the Segmentation toolbox in SPM), realigned to the first functional volume of each session, unwarped using a field map for phase correction, and slice-time corrected (Sladky et al., 2011). The MDEFT anatomical scan was coregistered to the mean unwarped functional image. All functional images were reoriented manually to ensure that the anterior commissure lay at coordinates $[0,0,0]$ and then spatially normalized to the Montreal Neurological Institute (MNI) EPI template. Functional images were first smoothed using a $4 \mathrm{~mm}^{3}$ full-width at half maximum 
(FWHM) Gaussian kernel. After estimating first-level models, contrast images were smoothed again using a $7 \mathrm{~mm}$ FWHM kernel, so that the final images were smoothed to $\sim 8 \mathrm{~mm}$.

\section{Statistical analysis}

For each participant, general linear models (GLMs) were used to model BOLD signals during performance of the stimulation-seeking task. The first-level models included the following regressors, convolved with the SPM synthetic hemodynamic response function:

Categorical analysis. This analysis was performed to examine differences in brain activity when choosing MES-associated and nonMES-associated stimuli, according to MES preference. Investigation of encoding of the points value of stimuli was used as an internal localizer to compare how encoding of economic reward related to encoding of MES association. Specifically, we examined BOLD responses during the decision period using a boxcar function time-locked to cue onset (duration, $1.5 \mathrm{~s}$ ), with the points value of the chosen CS, and whether a CS + or CSwas chosen on each trial ( +1 or -1 , respectively) added as parametric modulators. Additional regressors representing shock receipt (stick function with duration $0 \mathrm{~s}$ at actual time of shock delivery) and omission trials (if any; duration $1.5 \mathrm{~s}$ from time of trial onset) were also added to the model. Trials were modeled as compound events, as the timing of our design meant that it was unlikely to be possible to reliably separate out anticipation and delivery responses to different trial events.

Trial-by-trial computational analysis. This analysis was performed to relate trial-by-trial variation in the modeled internal probability or SAV of CSs $\left(S A V_{\mathrm{CS}}\right)$ to encoding of economic value. As in the categorical analysis, each trial was modeled as a compound event during the decision period, time-locked trial to cue onset (duration, $1.5 \mathrm{~s}$ ), with the points value of the chosen CS and modeled SAV of the chosen CS as parametric modulators. Additional regressors representing shock receipt and omission trials were also added to the model as for the first analysis.

Sensory prediction error analysis. This third analysis was performed to test the hypothesis is that sensory prediction errors (SPEs) would have opposing signs in MES-seeking versus MES-avoiding individuals. Trials were therefore modeled with onsets at the start of the inter-stimulus interval (ISI) period (duration, $2.5 \mathrm{~s}$ ), with parametric modulators of the points value just received, whether a CS+ or CS - was chosen, and the SPE for that trial, calculated according to Equation 4 (i.e., $S P E_{t}=S-$ $S A V_{C S, t-1}$; where $S$ is 1 when a shock was delivered and 0 otherwise, and $S A V_{C S, t-1}$ is the SAV of the chosen CS at the time of choice). Regressors representing shock receipt and omission trials were also added to the model as for the first analysis.

All analyses were corrected for serially correlated errors by fitting a first-order autoregressive process [AR(1)], and a high-pass filter $(1 / 128 \mathrm{~s})$ was used to attenuate linear scanner drift in low-frequency components. Six movement parameters generated during image realignment were also included in each model as regressors of no interest.

First-level contrasts were created through linear combinations of the resulting $\beta$ images. These contrast images were analyzed at the group level with one-sample $t$ tests, with individual $\theta$ values added as a covariate. A cluster-forming threshold of $p<0.001$ (uncorrected) was applied to statistical tests at the group level, followed by cluster-level familywise error (FWE) correction at $p<0.05$. Small-volume correction (SVC) at the cluster level was used in a priori regions of interest (ROIs). For the reported conjunction analyses, we used a conservative approach that required that each voxel exceed threshold in each of the examined contrasts separately (Nichols et al., 2005).

ROIs were identified on the basis of our hypotheses (see Introduction), in combination with prior functional imaging studies that examined differences in neural processing between groups of high and low selfreported sensation-seekers during decision-making and emotional processing tasks (Abler et al., 2006; Joseph et al., 2009; Freeman and Beer, 2010; Straube et al., 2010; Kruschwitz et al., 2012). These ROIs consisted of the vmPFC, vS, and insula.

The vmPFC ROI was defined using the MNI coordinates $[x, y, z]$ and extent estimate generated from a meta-analysis of neural valuation processes (right: 4.27, 35.18, 11.82; left: $-7.29,38,-10.57$; spheres with 12 $\mathrm{mm}$ radii; Levy and Glimcher, 2012). The vS ROI was also defined using estimates from the functional imaging literature, following Engelmann et al., 2015 ("nucleus accumbens": $\pm 9,9,-8$; "ventral caudate": $\pm 10,15,0$; spheres with $6 \mathrm{~mm}$ radii; anatomically derived coordinates previously found to be consistent with functional connectivity-based parcellation of the striatum; Di Martino et al., 2008). The insula ROI was defined anatomically using the automated anatomical labeling atlas (TzourioMazoyer et al., 2002) in the WFU PickAtlas toolbox (Wake Forest University School of Medicine). Where indicated, mean parameter estimates across ROIs were extracted using MarsBaR r0.44 (Brett et al., 2002).

For illustration purposes, all images are thresholded at $p<0.001$, uncorrected, and overlaid on the average normalized anatomical image.

\section{Results}

\section{Behavioral data}

In-scanner behavior

First, we confirmed that task performance was significantly related to sensation-seeking personality. To do this, we fit a simple computational model (see Materials and Methods; Fig. 1B) to choices from all trials to obtain an estimate for the value (in points) that each participant assigned to the opportunity to receive the MES $(\theta)$. When considering behavior inside the scanner, there was a significant positive relationship between $\theta$ value and self-reported sensation-seeking on a questionnaire measure (the Zuckerman Sensation-Seeking Scale version V, Revised, total score; Zuckerman, 1994; Gray and Wilson, 2007; $r=0.391$, $p<0.05$; Fig. $1 C$ ), replicating our previous findings (Norbury et al., 2015). Higher self-reported sensation-seekers assigned greater economic value to the opportunity to experience the MES, a sensation all participants endorsed as being "stimulating but not painful" (see Materials and Methods).

There was also a significant negative relationship between relative reaction time for MES-associated $(\mathrm{CS}+)$ versus nonassociated (CS - ) stimuli and $\theta$ value $(r=-0.543, p<0.01$; Fig. $1 D)$. Individuals who assigned a high value showed a relative speeding of responses for CS + stimuli, while those who assigned a lower value demonstrated relative slowing for $\mathrm{CS}+$ stimuli. This finding, again replicating our previous observations, is consistent with the hypothesis that MES-associated stimuli were considered appetitive, and thus evoked approach-like speeded reactions, in individuals who sought out such stimuli (stimulation-seekers), but were somewhat aversive and thus evoked avoidance-like slowed reactions in people who avoided them (stimulationavoiders; Norbury et al., 2015).

To test for possible time-on-task effects, $\theta$ estimates derived separately from behavior from each of the three blocks performed inside the scanner were entered into a repeated-measures model with the within-subjects factor of block. There was no overall effect of block number on $\theta$ values $\left(F_{(2,50)}=0.432, \eta_{\mathrm{p}}^{2}=\right.$ $0.017, p>0.1)$, suggesting no overall tendency across participants to reduce choice of MES-associated stimuli with decreasing stimulus novelty. When stimulation-seeking group $(\theta>0$ or $<0$, across all trials) was added to the model as a between-subjects factor, the block ${ }^{*}$ SS group interaction was also nonsignificant $\left(F_{(2,48)}=0.513, \eta_{\mathrm{p}}^{2}=0.021, p>0.1\right.$; Fig. $\left.1 E\right)$.

\section{Prescan behavior}

We then checked whether any observed behavioral differences during the imaging session could be attributed to differences in learning before entering the scanner.

First, we investigated whether there was any evidence of differences in learning about the points values of each CS between stimulation seekers and avoiders. Data from the prescan acquisition (points learning) phase were entered into a repeated- 
measures ANOVA to test whether the points value of the CSs (four levels: 25, 50, 75, and 100) was reflected in their overall proportionate choice. There was a significant main effect of points value on proportionate choice of $\operatorname{CSs}\left(F_{(3,75)}=92.946\right.$, $\left.\eta_{\mathrm{p}}^{2}=0.788, p<0.001\right)$, indicating that participants were indeed aware of the points value of each CS. Simple effects analysis by pairwise comparison revealed a significant difference in choice between each respective points level (all $p<0.001$; Fig. $1 F$ ). When including stimulation-seeking group as a between-subjects factor in the model, there was no significant interaction between group and points value $\left(F_{(3,72)}=0.649, \eta_{\mathrm{p}}^{2}=0.026, p>0.1\right)$, which is consistent with a lack of difference in learning of CSpoints associations according to stimulation preference.

Similarly, we checked for any evidence of differences in learning about the CS-MES associations between those who sought and those who avoided additional sensory stimulation. Learning of CS-MES association was probed using a repeated-measures model of "shock knowledge" based on VAS ratings of each CS [ratings on a VAS ranging from "no chance of shock" $(-300)$ to "chance of shock" $(+300)]$. There was a significant difference in mean VAS ratings for CS+ (MES-associated) compared with CS - (non-MES-associated) stimuli (significant main effect of CS type: $F_{(1,25)}=24.202, \eta_{p}^{2}=0.492, p<0.001$; Fig. $\left.1 G\right)$. Again there was no interaction between CS type and stimulation-seeking group $\left(F_{(1,24)}=0.304, \eta_{\mathrm{p}}^{2}=0.012, p>0.1\right)$, which is consistent with a lack difference in explicit knowledge about CS-MES associations between individuals who sought out versus individuals who tended to avoid MES-associated stimuli.

We also examined whether there were associations between stimulation-seeking and "liking" ratings of both the CS and MES itself in the prescan data. There was a significant positive association between change in VAS liking rating of MES-associated CSs following introduction of the mild electric stimuli and the value assigned to opportunity to receive the MES calculated from behavioral data (i.e., $\theta$ ). Thus individuals who assigned a higher value tended to increase their liking ratings, while individuals who assigned a lower value tended to decrease liking ratings $(r=$ 0.489, $p<0.05$; Fig. $1 H$ ) after CSs were associated with shocks.

\section{Evaluation of model fit}

To evaluate model fit, we first used model comparison to determine that our model explained participants' choice behavior significantly better than chance (likelihood ratio test across all subjects, $\chi_{52}^{2}=3833, p<1 \mathrm{e}-10$, for subjects individually all $p$ 's $<$ 0.0001; Daw, 2011). We also determined that our model fit better than a simpler nested model where participants chose only according to the economic (points) value of stimuli (i.e., $\theta=0$ for all subjects; likelihood ratio test for all subjects, $\chi_{(26)}^{2}=1849, p<$ 1e-10, significantly better fit at the individual level for 18 of 26 subjects, $p<0.05$ ). The mean pseudo- $r^{2}$ statistic (a descriptive measure of model fit appropriate for comparing between studies; Camerer and Ho, 1999) for all participants was 0.354 ( \pm 0.174$)$. There was no evidence that amount of variance explained by the model differed between stimulation-seekers and stimulationavoiders (mean pseudo- $r^{2}=0.369 \pm 0.199,0.348 \pm 0.168$, respectively; $p>0.1$; note this statistic applies to variance explained across all trials, including early on when performance and model fit are expected to be at chance levels).

There was no relationship between $\theta$ and $\beta$ (the softmax choice stochasticity parameter) estimates $(r=0.063, p>0.1)$, suggesting that stimulation seekers and avoiders were equally value-driven in their choices (mean $\beta$ : stimulation-seekers, $0.078 \pm 0.028$; stimulation-avoiders, $0.072 \pm 0.047 ; p>0.1$ ).
This also suggests that the model fits equally well for stimulation seekers versus avoiders as nonexplained choice variance is partitioned into the $\beta$ parameter for this kind of model. For the trialby-trial analysis, there was a moderate relationship between $\theta$ estimates and the learning rate parameter $\alpha(r=0.444, p=$ 0.023 ), suggesting those with higher $\theta$ values (stimulationseekers) may have learned a little faster about the shock association of individual CSs than stimulation-avoiders, perhaps as they were more keen to sample MES-associated stimuli (mean $\alpha$ : stimulation-seekers, $0.18 \pm 0.107$; stimulation-avoiders, $0.046 \pm$ 0.060; independent-samples $t=-3.994, p=0.001$ ).

\section{Reliability of behavior between prescreen and in-scanner testing} Across-session reliability of $\theta$ estimates [as measured by intraclass correlation coefficient (ICC); Shrout and Fleiss, 1979; McGraw and Wong, 1996] was found to be good for relative (rank) agreement (two-way mixed-model, average-measures ICC, 0.618), with a slightly lower value for absolute agreement (ICC, 0.531). This was due to a tendency for the value participants assigned to the opportunity to experience the MES to decrease between prescreening and scanning sessions (at recruitment: $\theta>0, N=19 ; \theta<0, N=7$; from scan day data: $\theta>0$, $N=8 ; \theta<0, N=18$ ). Formally, if $\theta$ estimates were contrasted between prescreen and scan-day sessions, there was a significant decrease in mean $\theta$ value across all subjects for trials completed inside the scanner (main effect of session: $F_{(1,25)}=12.164, \eta_{\mathrm{p}}^{2}=$ $0.327, p=0.002$; mean $\theta$ on prescreen, $3.9 \pm 14$; mean $\theta$ from scan trials, $-8.4 \pm 19)$.

This might reflect an effect of the unusual sensory environment of the scanner. In particular, for participants for whom this was their first ever MRI, there might have been decreased motivation for additional "intense and unusual" sensory stimulation in the unusual, dark, and loud environment of the scanner, as this experience may be sufficiently intense and unusual in and of itself. This explanation is in line with previous observations of differences in sensory processing between laboratory and MR environments (Ellerbrock and May, 2015).

\section{Functional imaging data}

Categorical analysis

We initially performed a simple categorical analysis to ascertain whether regional BOLD signal was sensitive to choice of CS+ (MES-associated) versus CS- (non-MES-associated) stimuli across all participants, during the decision period.

Choice of MES-associated versus non-MES-associated stimuli. There was significantly greater BOLD activity when choosing $\mathrm{CS}+$ as opposed to CS - stimuli in the right superior frontal gyrus (SFG), right and left inferior parietal lobule (IPL), right and left insula, right thalamus, and right inferior frontal gyrus (IFG; Table 2). Across all participants, there was no significant increase in activity to CS + versus CS - stimuli in either the vS or vmPFC ROIs.

To investigate the effects of individual differences in preference for MES-associated stimuli (as indexed by $\theta$ ) on brain activity when choosing between MES-associated and non-MES-associated stimuli, we next examined the effects of the continuous $\theta$ value covariate on the CS + versus CS- contrast. When examining the positive contrast at the whole-brain level, we found that there was a significant positive relationship (i.e., greater activity when choosing CS+ stimuli in individuals with greater $\theta$ value) in the left parahippocampal gyrus, the left precuneus, and the left cerebellum (Table 3 ).

Analysis of activity in our predefined vmPFC and vS ROIs revealed that $\theta$ value was significantly positively correlated with 
Table 2. Whole-brain analysis for choice of CS+ (MES-associated) versus CS(non-MES-associated) stimuli

\begin{tabular}{|c|c|c|c|c|c|c|c|}
\hline \multirow[b]{2}{*}{$p$} & \multirow[b]{2}{*}{$k$} & \multirow[b]{2}{*}{$t$} & \multirow[b]{2}{*}{ Z } & \multicolumn{3}{|c|}{ MNI coordinates } & \multirow[b]{2}{*}{ Region } \\
\hline & & & & $x$ & $y$ & $Z$ & \\
\hline \multirow[t]{3}{*}{$<0.001$} & 798 & 7.48 & 5.32 & 57 & -28 & 31 & Right IPL \\
\hline & & 7.18 & 5.20 & 63 & -34 & 34 & Right IPL \\
\hline & & 6.66 & 4.97 & 42 & -19 & 16 & Right insula \\
\hline \multirow[t]{3}{*}{0.001} & 227 & 7.13 & 5.17 & -51 & -31 & 28 & Left IPL \\
\hline & & 6.58 & 4.93 & -63 & -25 & 25 & $\begin{array}{l}\text { Left supramarginal } \\
\text { gyrus }\end{array}$ \\
\hline & & 4.09 & 3.53 & -54 & -46 & 34 & $\begin{array}{l}\text { Left supramarginal } \\
\text { gyrus }\end{array}$ \\
\hline \multirow[t]{3}{*}{$<0.001$} & 551 & 6.06 & 4.67 & 9 & 8 & 70 & Right SFG \\
\hline & & 5.36 & 4.30 & 9 & 23 & 43 & Right MFG \\
\hline & & 4.62 & 3.87 & 6 & 29 & 52 & Right SFG \\
\hline \multirow[t]{3}{*}{$<0.001$} & 299 & 6.00 & 4.64 & -27 & 26 & 1 & Left insula \\
\hline & & 5.97 & 4.63 & -30 & 20 & 10 & Left insula \\
\hline & & 5.18 & 4.20 & -48 & -1 & 7 & Left insula \\
\hline \multirow[t]{3}{*}{$<0.001$} & 436 & 5.98 & 4.64 & 30 & 26 & 4 & Right insula \\
\hline & & 5.82 & 4.55 & 33 & 23 & -5 & Right insula \\
\hline & & 5.55 & 4.41 & 27 & 20 & -14 & Right insula \\
\hline \multirow[t]{2}{*}{0.045} & 91 & 5.86 & 4.57 & 42 & 5 & 40 & Right IFG \\
\hline & & 4.00 & 3.47 & 51 & 5 & 52 & Right MFG \\
\hline \multirow[t]{3}{*}{0.016} & 122 & 5.00 & 4.10 & 12 & -13 & 4 & Right thalamus \\
\hline & & 4.09 & 3.53 & 9 & -22 & -5 & Right thalamus \\
\hline & & 3.71 & 3.27 & 12 & 2 & 10 & Right thalamus \\
\hline
\end{tabular}

$k$, Cluster size. All data whole-brain FWE cluster-level corrected (with an initial cluster-forming threshold of $p<$ 0.001).

Table 3. Whole-brain and ROI analyses for effects of $\theta$ value on BOLD when choosing MES-associated (CS+) versus non-MES-associated (CS-) stimuli

\begin{tabular}{lllllllllll}
\hline & & & & & \multicolumn{3}{l}{ MNI coordinates } & Significance \\
\cline { 5 - 7 } & $k$ & $t$ & $Z$ & $x$ & $y$ & $z$ & correction Region \\
\hline
\end{tabular}

Positive $\theta$

covariate

$\begin{array}{llllllll}<0.001 & 9300 & 11.7 & 6.68 & -33 & -40 & -11 & \text { WB }\end{array}$ $\begin{array}{llllll}9.35 & 6.01 & -27 & -37 & -20 & \text { WB }\end{array}$

Left PHG

Left fusiform

$\begin{array}{llllll}8.88 & 5.86 & -54 & -1 & -20 & \text { WB }\end{array}$

$<0.001$

$\begin{array}{lllllll}685 & 6.66 & 4.97 & -33 & -82 & 37 & \text { WB }\end{array}$

$\begin{array}{llllll}6.44 & 4.86 & -21 & -79 & 43 & W B\end{array}$

$\begin{array}{lllll}5.86 & 4.57 & -42 & -73 & 34\end{array} \mathrm{WB}$

$\begin{array}{lllllll}114 & 4.80 & 3.98 & -33 & -79 & -32 & \text { WB }\end{array}$

$\begin{array}{lllllll}3.82 & 3.34 & -51 & -61 & -32 & \text { WB }\end{array}$

$\begin{array}{llllll}3.59 & 3.18 & -48 & -52 & -41 & \text { WB }\end{array}$

$\begin{array}{lllllllll}<0.001 & 340 & 7.55 & 5.35 & -12 & 47 & -8 & \text { SVC }\end{array}$

$\begin{array}{llllll}7.04 & 5.14 & -12 & 38 & -11 & \text { SVC }\end{array}$

$\begin{array}{llllll}6.62 & 4.95 & 0 & 35 & -11 & \text { SVC }\end{array}$

$\begin{array}{llllllll}0.006 & 21 & 5.62 & 4.44 & 6 & 20 & -2 & \text { SVC }\end{array}$

$\begin{array}{llllllll}0.005 & 26 & 5.45 & 4.36 & -9 & 20 & -5 & \text { SVC }\end{array}$

$\begin{array}{llllll}4.43 & 3.75 & -3 & 11 & -5 & \text { SVC }\end{array}$

Negative $\theta$

covariate

0.012

$\begin{array}{lllllll}130 & 5.74 & 4.51 & 33 & 23 & -5 & \text { WB }\end{array}$

$\begin{array}{llllll}3.97 & 3.45 & 36 & 26 & 10 & \text { WB }\end{array}$

0.050 gyrus

Left mid temporal gyrus Left precuneus Left precuneus Left precuneus Left cerebellum Left cerebellum Left cerebellum Left vmPFC Left vmPFC Central vmPFC Right caudate Left caudate Left caudate

Right insula

Right IFG

Left insula Left insula

$k$, Cluster size; WB, Whole-brain FWE cluster-level corrected (with an initial cluster-forming threshold of $p<0.001$ ) SVC, small-volume corrected (FWE cluster-level corrected within ROI); PHG, parahippocampal gyrus.

responses in both regions. Specifically, there was significantly greater activity when choosing CS + compared with CS - stimuli in individuals with greater $\theta$ value (stimulation-preferring participants) in the left vmPFC and the head of the caudate bilaterally (Fig. 2A; Table 3 ). Individuals who assigned a positive value $(\theta)$ to the opportunity to receive the MES showed greater re-

sponse when choosing MES-associated relative to non-MESassociated stimuli in these regions, while the converse pattern was observed in individuals with a negative $\theta$ value.

Positive correlations between activity in the vmPFC and vS when choosing CS + versus CS - stimuli and individual $\theta$ values remained strongly significant after rerunning the analysis with the exclusion of a potential outlier from the model $(\theta=46.6$; Fig. $2 A$; positive effect of $\theta$ covariate on $\mathrm{CS}+$ vs $\mathrm{CS}-$ contrast in vmPFC: peak voxel: $-12,47,-8 ; k=206, Z=4.48, p_{S V C}<$ 0.001 ; in left vS: peak voxel: $-9,20,-5 ; k=8, Z=3.88, p_{S V C}=$ 0.017, in right vS: peak voxel: $9,20,-5 ; k=14, Z=3.75$, $p_{S V C}=0.011$ ).

There was also a significant negative relationship between bilateral insula response when choosing CS + relative to CS - stimuli and $\theta$ value (Fig. 2B; Table 3). Individuals who assigned a negative value to opportunity to receive the MES showed increases in BOLD signal bilaterally in the insula when choosing MES-associated versus non-MES-associated CSs, whereas individuals who assigned a positive value to opportunity to receive the MES did not. There was also a nonsignificant trend toward negative modulation of BOLD signal by $\theta$ value in the right medial frontal gyrus (MFG; peak voxel: $6,26,43 ; k=73, Z=3.53$, $\left.p_{\mathrm{FWE}}=0.080\right)$.

Points (economic) value of chosen stimuli. The stimulationseeking task was designed such that the points value of the chosen CS could be used to identify brain regions where BOLD signal was correlated with signals of expected economic reward. Thus, we examined the results of the parametric contrast encoding the points value of the chosen stimulus during the decision period. The positive contrast revealed no significant clusters at the whole-brain level. However, application of the vmPFC ROI revealed a significant cluster in the left vmPFC (peak voxel: $-6,41$, $\left.-8 ; k=4, Z=3.20, p_{\mathrm{SVC}}=0.046\right)$, and a cluster that narrowly missed significance in the right vmPFC (peak voxel: $9,44,-8$; $\left.k=1, Z=3.10, p_{\mathrm{SVC}}=0.065\right)$. The vS ROI revealed no significant clusters. The negative contrast (increasing BOLD signal with decreasing points value of the chosen stimulus) revealed significant clusters at the whole-brain level bilaterally in the MFG and IFG, bilaterally in the IPL, and in the left insula (peak voxel: -30 , 23, $7 ; k=113, Z=4.74, p=0.027)$.

Notably, points (economic) values of chosen stimuli represented an orthogonal contrast to MES association following model estimation, as both regressors were derived from the same model (mean absolute $r$ value for pairwise correlation between regressors before model estimation, $0.09 \pm 0.07$; range, $0.001-0.233$ ).

Conjunction analysis: common coding of economic and MES reward in stimulation-seekers? We next tested our hypothesis that the opportunity to experience the intense sensory stimulus would be encoded in the same circuitry as the opportunity to gain points (monetary reward), according to preference for the sensory stimulation (our behavioral index of stimulation-seeking, $\theta$ ). To do this, we used a conjunction analysis to test whether brain regions where BOLD signal positively encoded the economic value of chosen CSs also showed increased signal when choosing MESassociated, relative to non-MES-associated, CSs. According to our prediction, this relationship should be evident only in individuals who assigned a positive value to the opportunity to experience the MES (i.e., stimulation-seekers, but not stimulation-avoiders).

Specifically, we used an inclusive mask generated by the positive contrast of the points value parametric modulator (see above) to test for differences in activity on the orthogonal CS+ versus $\mathrm{CS}-$ contrast with individual differences in $\theta$ value. Ac- 
A
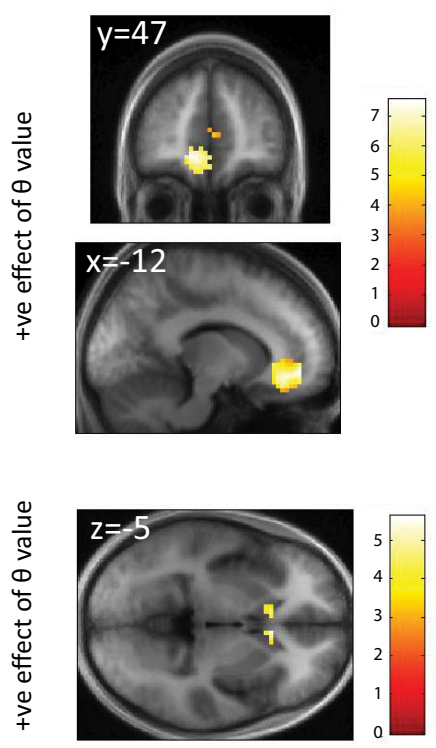

B

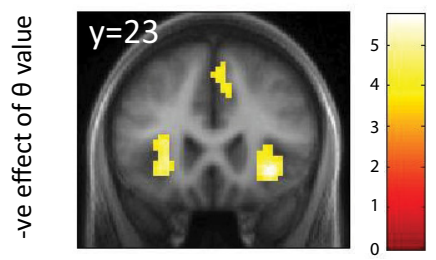

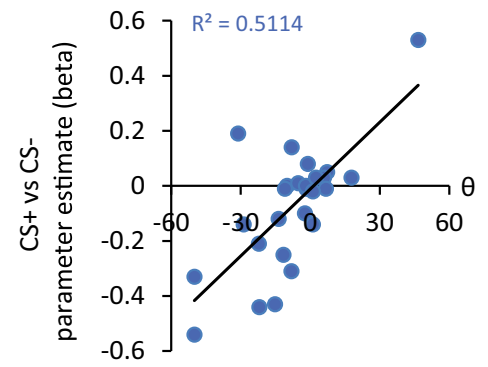
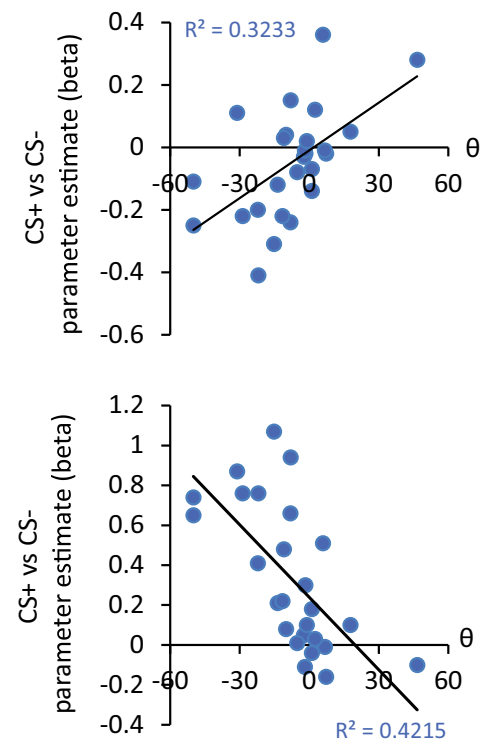

Figure 2. Effects of individual preference for the MES on BOLD signal when choosing MES-associated versus non-MESassociated stimuli in the vmPFC, vS, and insula. $A$, Significant positive effects of $\theta$ value (valued assigned to opportunity to receive the MES) on BOLD signal when choosing MES-associated (CS + ) as opposed to non-MES-associated (CS - ) stimuli in the left vmPFC and head of the caudate, bilaterally. B, Significant negative effect of $\theta$ value on BOLD signal when choosing CS + compared with $C S-$ stimuli in the insula, bilaterally. Color bars represent $t$ values. Scatter plots represent mean $\beta$ estimates extracted from CS + versus $C S$ - contrast images across whole a priori ROls $(\boldsymbol{A})$ and at peak voxel coordinates identified from the group-level contrast $(\boldsymbol{B})$, plotted against individual $\theta$ values.

tivity in the points value-sensitive vmPFC cluster was greater when choosing CS + stimuli in individuals who assigned greater value to opportunity to receive the MES (i.e., $\theta$ was significantly positively correlated with activity in this region; peak voxel: -6 , $44,-8 ; k=3, Z=4.88, p_{\mathrm{SVC}}=0.002$; Fig. $3 A$ ).

Further, activity in this points value-sensitive region was positively signed when choosing MES-associated (or CS + ) stimuli in stimulation-seekers $(\theta>0)$, but negatively signed in stimulationavoiders $(\theta<0$; significant difference between groups in parameter estimates from CS + versus CS - contrast when extracted from the peak voxel identified from the positive points value contrast, $t_{(22)}=-2.916, p=0.008$, independent samples $t$ test; Fig. 3A).

Conversely, when masking this contrast with areas that showed increased activity with decreasing points value of the chosen CS, $\theta$ value was significantly negatively correlated with activity bilaterally in the insula (right peak voxel: $-27,23,-2 ; k=60, Z=4.00, p_{\mathrm{SVC}}=$ 0.006 ; left peak voxel: $\left.33,23,-5 ; k=49, Z=4.51, p_{\mathrm{svC}}=0.010\right)$. Individuals with low $\theta$ values (stimulation avoiders) showed positive activity in a brain region negatively associated with economic value when choosing MES-associated stimuli, but individuals with high $\theta$ values (stimulation seekers) did not (significant differ- ence between groups in parameter estimates from CS + vs CS - contrast when extracted from the peak insula voxel identified from the negative points value contrast; $t_{(24)}=$ 2.609, $p=0.015$, independent samples $t$ tests; Fig. 3B).

Trial-by-trial computational analysis SAV of chosen stimuli. We next investigated whether information about the specific expected probability of receiving an MES, given choice of a particular CS, was encoded differently in stimulationseekers and stimulation-avoiders. To do this, we first created a simple computational model (see Materials and Methods) to derive each subject's internal probability estimate of receiving the MES at the time of CS choice [i.e., trial-by-trial variation in the SAV of each CS $\left(S A V_{\mathrm{CS}}\right)$; Fig. $4 A]$. These $S A V_{C S}$ values were then incorporated into the fMRI analysis to identify areas where these values correlated with BOLD signals. The positive contrast of trial-by-trial $S A V_{C S}$ value across the whole group revealed a significant cluster in the left insula (peak voxel: $-27,29,1 ; k=26$, $\left.Z=3.56, p_{\mathrm{SVC}}=0.020\right)$, with a trend in the right insula (peak voxel: $36,14,-11$; $k=11, Z=3.37, p_{\mathrm{SVC}}=0.064$; Fig. $4 A$ ). This suggests that, across all subjects, the modeled internal probability of receiving the MES was tracked in the insula during the choice period. The negative contrast revealed no significant clusters.

There was no evidence for a (linear) effect of $\theta$ value on the positive contrast at our significance threshold. To explore effects undetected by this analysis (e.g., due to nonlinear effects of $\theta$ value on BOLD signal), we used a two-sample $t$ test at the second level, with participants divided into stimulation-seekers $(\theta>0)$ and stimulationavoiders $(\theta<0)$.

This grouping was then used to explore differences between stimulation seekers and avoiders in coding of SAV $\left(S A V_{C S}\right)$, by contrasting activity between the two groups in brain regions previously found to be sensitive to $S A V_{C S}$ value on each trial. This analysis revealed significantly greater parameter estimates for the $S A V_{C S}$ contrast in stimulation-avoidant, compared with stimulation-seeking, individuals in the left insula (peak voxel: $\left.-33,23,-5 ; k=23, Z=3.81, p_{S V C}=0.028\right)$. Thus, there appeared to be significant positive coding of SAV in the left insula for stimulation-avoiders, but not stimulation-seekers. There were no significant clusters where activity was found to be greater for stimulation-seeking, compared with stimulation-avoiding, participants.

In this model, analysis of brain regions where BOLD signal was sensitive to the points value of the CS at the time of decision again revealed a significant left vmPFC cluster for the positive contrast (peak voxel: $-6,41,-8 ; k=15, Z=3.36$, $\left.p_{\text {SVC }}=0.024\right)$. Similarly, the negative contrast again revealed significant clusters at the whole-brain level in the IPL, IFG, 
A
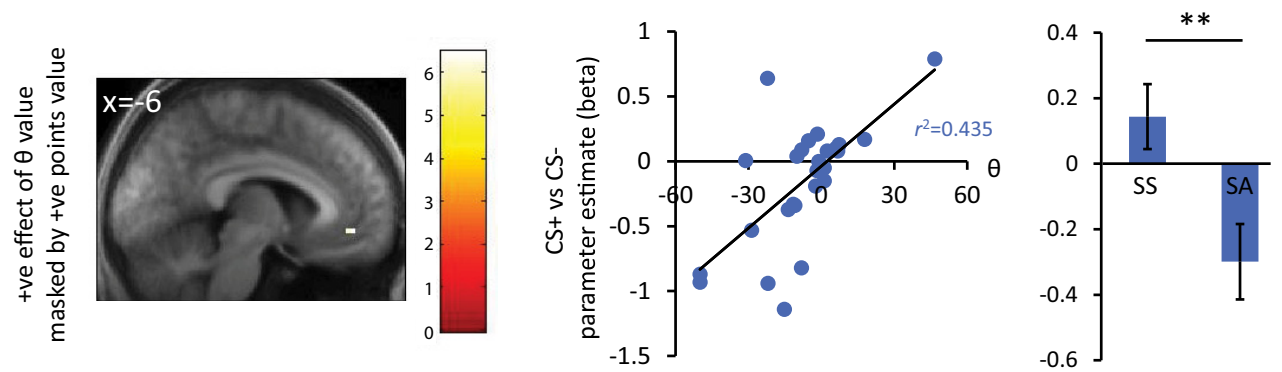

B
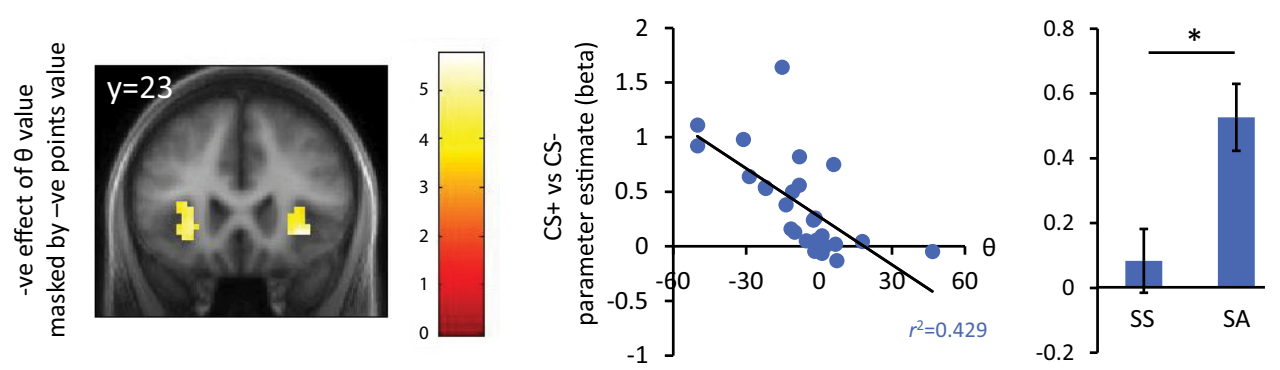

Figure 3. Common encoding of the economic and MES-association of choice stimuli in the vmPFC and insula, depending on MES preference. A, Conjunction analysis revealed that a region in the vmPFC cluster identified as showing increasing activity with increasing points value of chosen CSs also showed positive modulation by individual $\theta$ value when choosing MES-associated (as opposed to non-MES-associated) stimuli. $\boldsymbol{B}$, Conjunction analysis revealed that insula clusters identified as showing increasing activity with decreasing points value of chosen CSs also showed negative modulation according to $\theta$ value when choosing MES-associated (as opposed to non-MES-associated) stimuli. Color bars represent $t$ values. Scatter plots and bar charts show individual parameter estimates from the $C S+$ versus $C S$ - contrast, extracted at peak voxels identified from the positive $(\boldsymbol{A})$ and negative $(\boldsymbol{B})$ points value group-level contrasts, plotted against $\theta$ values. SS, Stimulation-seeking $(\theta>0)$; SA, stimulation-avoiding $(\theta<0)$. Error bars represent SEM. ${ }^{* *} p=0.008,{ }^{*} p=0.015$.

and MFG, and bilaterally in the insula. Again, points value represented an orthogonal contrast to $S A V_{\mathrm{CS}}$ following model estimation, as both regressors were derived from the same model (mean absolute $r$ value for pairwise correlation between regressors before model estimation, $0.08 \pm 0.04$; range, $0.006-0.153)$.

Conjunction analysis. Similarly to the categorical analysis, we wanted to test whether modeled information about the precise probability of receiving the MES $\left(S A V_{\mathrm{CS}}\right)$ was encoded in the same way as positive economic reward, selectively in stimulationseeking individuals. Conversely, we expected that this information may be encoded in the same way as negative economic information in stimulation-avoiders. We therefore examined whether, during the decision period, brain regions where BOLD signals were significantly positively associated with the economic (points) value of chosen stimuli also showed significant positive encoding of the modeled $S A V_{\mathrm{CS}}$, and related this to stimulationseeking status.

When masked by regions significantly negatively encoding the points value of the chosen CS (i.e., exhibiting increasing activation with decreasing points value), there was again greater activity in stimulation-avoiders than in stimulation-seekers on the $S A V_{C S}$ contrast in the left insula (peak voxel: $-30,23,-5 ; k=21, Z=$ $3.79, p_{\mathrm{SVC}}=0.036$ ). There was positive coding of modeled probability of receiving an MES in regions tracking negative economic value in stimulation-avoiders, but not in stimulation-seekers (significant difference between groups in parameter estimates from the $S A V_{C S}$ regressor when extracted from the peak voxel identified from the negative points-value contrast, $t_{(20)}=2.754$, $p=0.012$, independent samples $t$ test; Fig. $4 A$ ).

Individuals with low preference for intense sensory stimulation thus appear to track the probability of receiving MES in the same way as receiving low economic value (fewer points), whereas stimulation-seekers do not. This is despite the fact that we found no evidence for a difference in learning about MESpredicting versus non-MES-predicting stimuli stimulationseekers versus stimulation-avoiders, prior to entering the scanner.

Analysis of activity on the $S A V_{C S}$ contrast in the peak voxel identified from the positive points-value contrast (i.e., the peak voxel across all subjects exhibiting increasing activation with increasing points value, located in the left vmPFC) revealed a nonsignificant trend toward greater parameter estimates in stimulation-seekers, as opposed to stimulation-avoiders (independent samples $t$ test, $p=0.052$; Fig. $4 B$ ).

\section{SPE analysis}

Finally, we investigated whether SPE signals might be oppositely encoded in high versus low stimulation-seeking individuals. Specifically, we wanted to test the hypothesis that actual receipt of the probabilistic MES represents a better-than-expected outcome for stimulation seekers, but worse-than-expected outcome for stimulation-avoiders.

First, we investigated whether BOLD signals in any brain region were significantly associated with the trial-by-trial prediction error signals generated using our computational model, during the period where participants either did or did not receive the MES (see Materials and Methods). Across the whole group, we found no significant clusters either at the whole-brain level or in our prespecified ROIs for the positive SPE contrast. However, we detected significant negative encoding of SPEs in clusters in the IPL/superior temporal gyrus and SFG (Table 4). This suggests that, overall, our participants negatively coded imperfectly predicted receipt of the MES (equivalent to coding $S=-1$ vs $S=0$, instead of $S=1$ vs $S=0$, in Eq. 4; Materials and Methods). This is unsurprising, given that the majority of our participants as- 
A
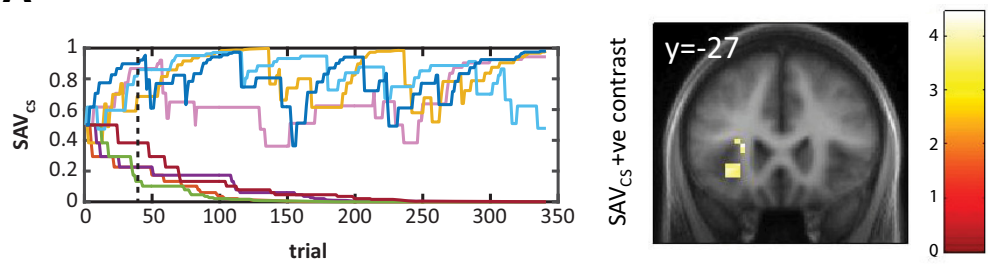

\section{B}

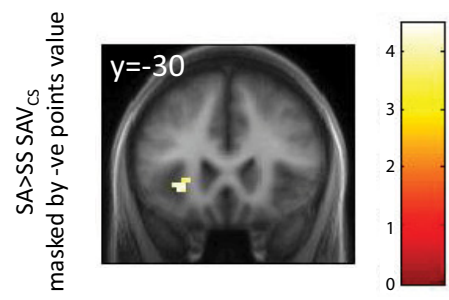

C

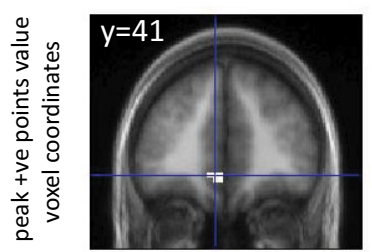

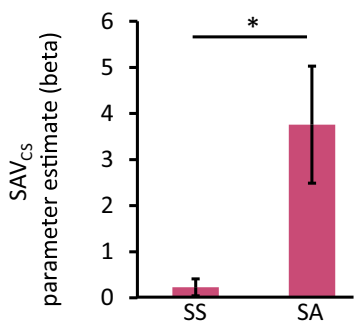

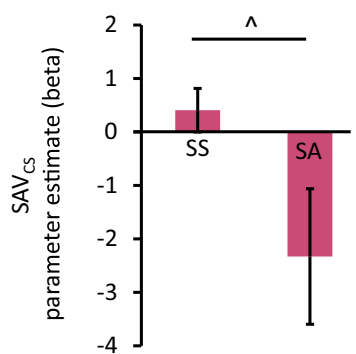

Figure 4. Common encoding of modeled MES-predictedive value and negative economic value in low stimulation-seekers. $\boldsymbol{A}$, Plot of modeled internal probability of receiving the MES for each $C S\left(S A V_{C S}\right)$, for a representative participant (left). Across all participants, the SAV of the chosen CS on each trial was represented in BOLD signal in the right insula (right). $\boldsymbol{B}$, Conjunction analysis revealed that an insular region identified as showing increasing activity with decreasing economic value of chosen stimuli showed positive coding of $S A V_{C S}$ in stimulation-avoiding, but not stimulation-seeking, individuals, during the decision period. $\boldsymbol{C}$, BOLD signal extracted from the peak voxel identified from the positive points-value contrast (i.e., a region significantly positively encoding the economic value of chosen (Ss, located in the vmPFC) revealed marginally greater encoding of SAV in stimulationseekers, compared with stimulation-avoiders. Color bars represent $t$ values. Bar charts represent parameter estimates extracted from the SAV contrast images, extracted at peak insula voxel coordinates from the negative points-value contrast $(\boldsymbol{A})$ and the positive points-value contrast $(\boldsymbol{B})$. SS, Stimulation-seeking $(\theta>0)$; SA, stimulation-avoiding $(\theta<0)$. Error bars represent SEM. ${ }^{*} p=0.012, \wedge p=0.052$.

Table 4. Whole-brain analysis for SPE signals (negative contrast)

\begin{tabular}{|c|c|c|c|c|c|c|c|}
\hline \multirow[b]{2}{*}{$p$} & \multirow[b]{2}{*}{$k$} & \multirow[b]{2}{*}{$t$} & \multirow[b]{2}{*}{ Z } & \multicolumn{3}{|c|}{ MNI coordinates } & \multirow[b]{2}{*}{ Region } \\
\hline & & & & $x$ & $y$ & $z$ & \\
\hline \multirow[t]{2}{*}{0.002} & \multirow[t]{2}{*}{202} & 6.34 & 4.81 & 48 & -64 & 40 & Right IPL \\
\hline & & 4.42 & 3.74 & 57 & -61 & 25 & Right STG \\
\hline \multirow[t]{3}{*}{0.013} & \multirow[t]{3}{*}{135} & 4.67 & 3.90 & 24 & 32 & 52 & Right SFG \\
\hline & & 4.37 & 3.71 & 15 & 35 & 55 & Right SFG \\
\hline & & 4.34 & 3.69 & 33 & 17 & 58 & Right SFG \\
\hline
\end{tabular}

k, Cluster size; STG, superior temporal gyrus. All data whole-brain FWE cluster-level corrected (with an initial clusterforming threshold of $p<0.001$ ).

signed a negative value to opportunity to receive the MES inside the scanner.

We next tested our hypothesis that SPEs might be positively signed in stimulation-seekers (where receipt of the MES represents a better-than-predicted outcome), but negatively signed in stimulation-avoiders (where receipt of the MES represents a worse-than-predicted outcome).

Using sensitivity to SPE signals across the whole group (Table 4) as a mask, we found greater BOLD signal in response to SPEs in stimulation-seekers (compared with stimulation avoiders) in a cluster in the left posterior cingulate cortex (PCC), although this cluster did not survive correction for multiple comparisons at the whole-brain level. As the PCC did not constitute an a priori ROI for this study (although previously shown to encode reward prediction errors; McCoy et al., 2003; Rutledge et al., 2010), we applied an exploratory a posteriori correction to this contrast, correcting for the search volume of the wholegroup mask (subthreshold PCC, plus IPL and SFG clusters as recorded in Table 4). Under this exploratory correction, SPEs appear to be coded positively for stimulation-seekers, but negatively coded for stimulation-avoiders, in the PCC (peak voxel: $-9,-55,28 ; k=11, Z=$ $4.14, p_{\mathrm{SVC}}=0.045$; Fig. 5).

\section{Discussion}

We investigated brain mechanisms during valuation of intense sensory experiences, and how these differ according to individual differences in preference for such experiences, by combining a novel behavioral paradigm with fMRI and computational analysis. Increased BOLD signal was observed in both the vmPFC and head of the caudate nucleus when choosing options associated with intense tactile stimulation (MES), as a linear function of the additional value participants assigned to opportunity to receive the MES (positive or negative; Fig. 2). Critically, we found evidence for common encoding of economic gain (points value) and MES association (sensory value) in the vmPFC of stimulation-seekers (Fig. 3). This finding is consistent with our hypothesis that intense sensory experiences are evaluated in the same way as other rewards in stimulation-seekers, using a common value currency (Levy and Glimcher, 2012; Clithero and Rangel, 2014).

We also found that fluctuation in expected MES probability (SAV) of chosen stimuli was tracked during the decision period in the left insula (Fig. 4). Both choice of MES-associated options, and the precise probability of receiving the MES given choice of a particular CS on a given trial, were associated with much stronger insula signal in stimulation-avoiders, compared with stimulation-seekers (who showed negligible insula activity in response to MES cues). It has previously been suggested that the insula has a role in tracking "salient" events (e.g., showing greater activation during anticipation of events of uncertain outcome), particularly but by no means exclusively in the case of unpleasant or aversive outcomes (Preuschoff et al., 2008; Menon and Uddin, 2010; Rutledge et al., 2010). Significantly, in stimulationavoiders, but not stimulation-seekers, probability of receiving the MES was encoded in the same way as a decrease in economic value. Thus we provide evidence consistent with both increased appetitive and reduced aversive anticipation of intense sensory stimulation in stimulation-seekers, as has been postulated by previous psychological theories of sensation-seeking personality (Zuckerman, 1994). 

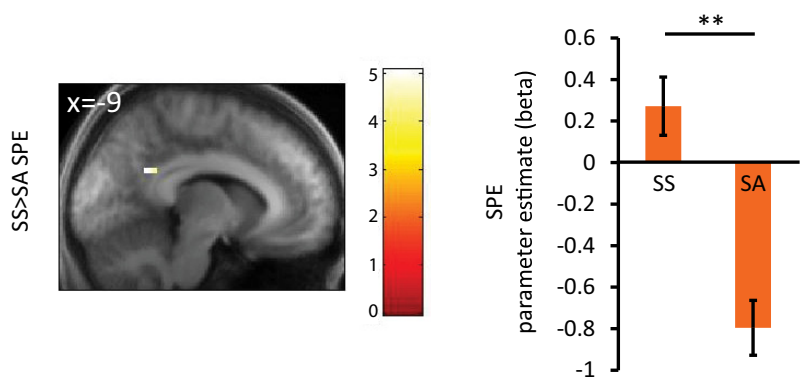

Figure 5. SPE signals may be positively signed for stimulation-seekers, but negatively signed for stimulation-avoiders, in the PCC. Color bar represents $t$ values. Bar chart represents parameter estimates extracted from SPE contrast images, extracted at peak voxel coordinates from the contrast between high and low stimulation-seekers, when masked by voxels sensitive to SPE signals across the whole group. SS, Stimulation-seeking $(\theta>0)$; SA, stimulationavoiding $(\theta<0)$. Error bars represent SEM. ${ }^{* *} p<0.001$.

Our findings in stimulation-avoidant individuals are in accordance with previous studies from the neuroeconomic literature probing the value coding of painful electric stimulation: notably a recent study by Winston et al. indicating that the amount participants were willing to pay to avoid electrical pain was negatively encoded by BOLD signal in the medial orbitofrontal cortex at the time of valuation (Winston et al., 2014). Berns et al. (2006) also found that anticipatory BOLD signal in the posterior insula was significantly greater in individuals who more strongly "dreaded" an upcoming painful shock (were willing to experience greater pain to shorten the delay until outcome). Interestingly, bilateral anterior insula activity has been found to predict whether or not a subsequent sensory stimulus (radiant heat) is perceived as painful, with greater prestimulus activity predicting greater likelihood that the stimulus will be rated as painful (Ploner et al., 2010), depending on contextual salience (Wiech et al., 2010). Thus, greater anticipatory insula activity in stimulation-avoiders when choosing MES-associated stimuli might relate to decreased "liking" ratings of the sensation of the MES by these individuals, despite all participants endorsing a description of the stimulus as "nonpainful." That we did not observe the same activity pattern in stimulation-seeking individuals is consistent with a previous observation of decreased aversionrelated physiological responses to cutaneous electrical stimulation in high, compared with low, self-reported sensationseekers (De Pascalis et al., 2007).

Finally, although preliminary in nature, the findings from our SPE analysis (Fig. 5) are consistent with previous observations of reward prediction error signals in the posterior cingulate cortex in both nonhuman primate electrophysiological and human fMRI data (McCoy et al., 2003; de Bruijn et al., 2009; Rutledge et al., 2010). However, an important caveat to this interpretation is that, by definition, prediction error signals covary significantly with the expected value of chosen options. As discussed in Materials and Methods, due to the timing of our design, we are unable to reliably separate out expectation from outcome-related signals in our analysis. Therefore, although BOLD signal did significantly covary with prediction error in the regions we identified, we cannot be sure that this was not driven by encoding of some other kind of value signal (see e.g. Clithero and Rangel, 2014, who identify PCC activity as being related to overall stimulus value across various decision-making tasks).

One limitation of the current study is that preference for the MES (our behavioral index of stimulation-seeking) de- creased between initial prescreening and fMRI testing sessions, resulting in a loss of data points from our functional imaging sample with (particularly intermediate) positive $\theta$ values. We have therefore tried, where possible, to use a continuous covariate approach in our analyses. However, it should be acknowledged that, even using this approach, our sensitivity to detect effects in the positive portion of the range of theta values is hampered. Thus, inferences about the direction of effects in stimulation-seekers must be treated with caution and will require independent replication. It is also possible that a lack of power explains why we did not find a significant conjunction between regions encoding positive economic value and SAV in stimulation-seeking individuals, while we did find the converse in stimulation-avoiders in the insula.

Further, by definition, there are systematic differences in total CS+ choice, and associated outcome frequencies, between stimulation-seeking and stimulation-avoiding individuals. This may lead to increased noise where choices are relatively less frequent, or conversely the presence of habituation effects only in individuals exhibiting more frequent choices of the MES. However, we attempted to ensure via our task design and number of trials that there will be sampling of all response types across all individuals.

Finally, an alternative explanation for the difference in preference for the MES between stimulation-seekers and stimulation-avoiders is a difference in somatosensory perception of the stimulus itself, rather than in sensation-seeking motivation per se (e.g., experiencing a ticklish sensation vs mild discomfort). In both this study and in two previously published samples, we have found that self-reported sensation-seeking personality accounts for $\sim 20 \%$ of variation in our MES value parameter $\theta$ (Norbury et al., 2015). Although this is toward the top end of previously reported correlations between behavioral and questionnaire measures of the related personality construct of impulsivity (Cyders and Coskunpinar, 2011), it may be that other factors, such as somatosensory differences, contribute significantly to variation in task performance. One previous study has examined subjective responses to cutaneous electric stimulation (ranging from just detectable, to unpleasant, to painful), and found that high and low self-reported sensation-seekers differed in their absolute sensory detection threshold, but not in the thresholds at which the stimulation was experienced as unpleasant or painful (De Pascalis et al., 2007). We attempted to minimize potential subpainful discomfort in our participants by using large EMG electrodes that produce a "vibratory" buzzing sensation (compare with pain studies which often use annular electrodes to evoke a more unpleasant, pin-prick-like sensation). However, we can provide no data that speaks to possible differences in somatosensory perception in our participants. Empirical testing in future studies would help address this issue.

Despite these limitations, we propose that the data summarized above contribute significantly to the understanding of the neural correlates of sensation-seeking behavior. Using a novel fMRI paradigm, we have demonstrated that intense sensory stimulation may activate brain areas encoding economic reward selectively in stimulation-seekers. This might help explain increased motivation for unusual sensory experiences, including physically risky and/or expensive leisure activities (Zuckerman, 1994; Roberti, 2004), and increased liking of the effects of recreational drugs in higher sensation-seeking individuals (Kelly et al., 2006; Fillmore et al., 2009; Zacny, 2010)—although it is important to note that the tendency to engage in these kinds of activities 
is likely to depend on a complex interplay of factors, both cognitive and socioeconomic (Hammelstein, 2004; Norbury and Husain, 2015). For example, it has recently been found the individual differences in anatomical structure in cognitive control circuitry are commonly associated with sensation-seeking personality and substance use (Holmes et al., 2016). We also found evidence that stimulation-seekers show decreased activity in a brain region implicated in "salience" detection (the insula) during anticipation of intense sensory stimulation, which may be related to their decreased avoidance reactions toward these stimuli. It will be important to investigate in future studies whether these findings hold in individuals with psychopathology associated with substance use disorders and extreme sensation-seeking.

\section{References}

Abler B, Walter H, Erk S, Kammerer H, Spitzer M (2006) Prediction error as a linear function of reward probability is coded in human nucleus accumbens. Neuroimage 31:790-795. CrossRef Medline

Berns GS, Chappelow J, Cekic M, Zink CF, Pagnoni G, Martin-Skurski ME (2006) Neurobiological substrates of dread. Science 312:754-758. CrossRef Medline

Brett M, Anton J-L, Valabregue R, Poline J-B (2002) Region of interest analysis using an SPM toolbox. Neuroimage 16. Paper presented at the 8th Internationnal Conference on Functional Mapping of the Human Brain. Sendai, Japan, June.

Büchel C, Morris J, Dolan RJ, Friston KJ (1998) Brain systems mediating aversive conditioning: an event-related fMRI study. Neuron 20:947-957. CrossRef Medline

Camerer C, Ho T-H (1999) Experience-weighted attraction learning in normal form games. Econometrica 67:827-874. CrossRef

Clithero JA, Rangel A (2014) Informatic parcellation of the network involved in the computation of subjective value. Soc Cogn Affect Neurosci 9:1289-1302. CrossRef Medline

Cyders MA, Coskunpinar A (2011) Measurement of constructs using selfreport and behavioral lab tasks: is there overlap in nomothetic span and construct representation for impulsivity? Clin Psychol Rev 31:965-982. CrossRef Medline

Daw ND (2011) Trial-by-trial data analysis using computational models. In: Decision making, affect, and learning: attention and performance XXIII, pp 3-39. Oxford: Oxford UP.

de Bruijn ER, de Lange FP, von Cramon DY, Ullsperger M (2009) When errors are rewarding. J Neurosci 29:12183-12186. CrossRef Medline

Deichmann R, Schwarzbauer C, Turner R (2004) Optimisation of the 3D MDEFT sequence for anatomical brain imaging: technical implications at 1.5 and $3 \mathrm{~T}$. Neuroimage 21:757-767. CrossRef Medline

De Pascalis V, Valerio E, Santoro M, Cacace I (2007) Neuroticism-anxiety, impulsive-sensation seeking and autonomic responses to somatosensory stimuli. Int J Psychophysiol 63:16-24. CrossRef Medline

Di Martino A, Scheres A, Margulies DS, Kelly AM, Uddin LQ, Shehzad Z, Biswal B, Walters JR, Castellanos FX, Milham MP (2008) Functional connectivity of human striatum: a resting state FMRI study. Cereb Cortex 18:2735-2747. CrossRef Medline

Ellerbrock I, May A (2015) MRI scanner environment increases pain perception in a standardized nociceptive paradigm. Brain Imaging Behav 9:848-853. CrossRef Medline

Engelmann JB, Meyer F, Fehr E, Ruff CC (2015) Anticipatory anxiety disrupts neural valuation during risky choice. J Neurosci 35:3085-3099. CrossRef Medline

Fillmore MT, Ostling EW, Martin CA, Kelly TH (2009) Acute effects of alcohol on inhibitory control and information processing in high and low sensation-seekers. Drug Alcohol Depend 100:91-99. CrossRef Medline

Freeman HD, Beer JS (2010) Frontal lobe activation mediates the relation between sensation seeking and cortisol increases. J Pers 78:1497-1528. CrossRef Medline

Gray JM, Wilson MA (2007) A detailed analysis of the reliability and validity of the sensation seeking scale in a UK sample. Personal Individ Differ 42:641-651. CrossRef

Haber SN, Knutson B (2010) The reward circuit: linking primate anatomy and human imaging. Neuropsychopharmacology 35:4-26. CrossRef Medline

Hammelstein P (2004) Faites vos jeux! Another look at sensation seeking and pathological gambling. Personal Individ Differ 37:917-931. CrossRef
Hayden BY, Heilbronner SR (2014) All that glitters is not reward signal. Nat Neurosci 17:1142-1144. CrossRef Medline

Holmes AJ, Hollinshead MO, Roffman JL, Smoller JW, Buckner RL (2016) Individual differences in cognitive control circuit anatomy link sensation seeking, impulsivity, and substance use. J Neurosci 36:4038-4049. CrossRef Medline

Joseph JE, Liu X, Jiang Y, Lynam D, Kelly TH (2009) Neural correlates of emotional reactivity in sensation seeking. Psychol Sci 20:215-223. CrossRef Medline

Kelly T, Robbins G, Martin C, Fillmore M, Lane S, Harrington N, Rush C (2006) Individual differences in drug abuse vulnerability: $d$-amphetamine and sensation-seeking status. Psychopharmacology (Berl) 189:17-25. CrossRef Medline

Knutson B, Adams CM, Fong GW, Hommer D (2001) Anticipation of increasing monetary reward selectively recruits nucleus accumbens. J Neurosci 21:RC159. Medline

Kruschwitz JD, Simmons AN, Flagan T, Paulus MP (2012) Nothing to lose: processing blindness to potential losses drives thrill and adventure seekers. Neuroimage 59:2850-2859. CrossRef Medline

Lawson RP, Seymour B, Loh E, Lutti A, Dolan RJ, Dayan P, Weiskopf N, Roiser JP (2014) The habenula encodes negative motivational value associated with primary punishment in humans. Proc Natl Acad Sci U S A 111:11858-11863. CrossRef Medline

Levy DJ, Glimcher PW (2012) The root of all value: a neural common currency for choice. Curr Opin Neurobiol 22:1027-1038. CrossRef Medline

Lissek S, Powers AS (2003) Sensation seeking and startle modulation by physically threatening images. Biol Psychol 63:179-197. CrossRef Medline

McCoy AN, Crowley JC, Haghighian G, Dean HL, Platt ML (2003) Saccade reward signals in posterior cingulate cortex. Neuron 40:1031-1040. CrossRef Medline

McGraw KO, Wong SP (1996) Forming inferences about some intraclass correlation coefficients. Psychol Methods 1:30-46. CrossRef

Menon V, Uddin LQ (2010) Saliency, switching, attention and control: a network model of insula function. Brain Struct Funct 214:655-667. CrossRef Medline

Nichols T, Brett M, Andersson J, Wager T, Poline JB (2005) Valid conjunction inference with the minimum statistic. Neuroimage 25:653-660. CrossRef Medline

Nitschke JB, Sarinopoulos I, Mackiewicz KL, Schaefer HS, Davidson RJ (2006) Functional neuroanatomy of aversion and its anticipation. Neuroimage 29:106-116. CrossRef Medline

Norbury A, Husain M (2015) Sensation-seeking: dopaminergic modulation and risk for psychopathology. Behav Brain Res 288:79-93. CrossRef Medline

Norbury A, Kurth-Nelson Z, Winston JS, Roiser JP, Husain M (2015) Dopamine regulates approach-avoidance in human sensation-seeking. Int J Neuropsychopharmacol 18:pyv041. CrossRef Medline

O'Doherty JP (2014) The problem with value. Neurosci Biobehav Rev 43: 259-268. CrossRef Medline

Olsen CM, Winder DG (2009) Operant sensation seeking engages similar neural substrates to operant drug seeking in C57 mice. Neuropsychopharmacology 34:1685-1694. CrossRef Medline

Ploner M, Lee MC, Wiech K, Bingel U, Tracey I (2010) Prestimulus functional connectivity determines pain perception in humans. Proc Natl Acad Sci U S A 107:355-360. CrossRef Medline

Preuschoff K, Quartz SR, Bossaerts P (2008) Human insula activation reflects risk prediction errors as well as risk. J Neurosci 28:2745-2752. CrossRef Medline

Roberti JW (2004) A review of behavioral and biological correlates of sensation seeking. J Res Personal 38:256-279. CrossRef

Rutledge RB, Dean M, Caplin A, Glimcher PW (2010) Testing the reward prediction error hypothesis with an axiomatic model. J Neurosci 30: 13525-13536. CrossRef Medline

Saunders JB, Aasland OG, Babor TF, de la Fuente JR, Grant M (1993) Development of the Alcohol Use Disorders Identification Test (AUDIT): WHO Collaborative Project on Early Detection of Persons with Harmful Alcohol Consumption-II. Addiction 88:791-804. CrossRef Medline

Shrout PE, Fleiss JL (1979) Intraclass correlations: uses in assessing rater reliability. Psychol Bull 86:420-428. CrossRef Medline

Skinner HA (1982) The drug abuse screening test. Addict Behav 7:363-371. CrossRef Medline 
Sladky R, Friston KJ, Tröstl J, Cunnington R, Moser E, Windischberger C (2011) Slice-timing effects and their correction in functional MRI. Neuroimage 58:588-594. CrossRef Medline

Speer ME, Bhanji JP, Delgado MR (2014) Savoring the past: positive memories evoke value representations in the striatum. Neuron 84:847-856. CrossRef Medline

Spielberger CD, Gorsuch RL, Lushene RE (1970) The state-trait anxiety inventory: test manual for form X. Palo Alto, CA: Consulting Psychologists.

Straube T, Preissler S, Lipka J, Hewig J, Mentzel HJ, Miltner WH (2010) Neural representation of anxiety and personality during exposure to anxiety-provoking and neutral scenes from scary movies. Hum Brain Mapp 31:36-47. Medline

Tzourio-Mazoyer N, Landeau B, Papathanassiou D, Crivello F, Etard O, Delcroix N, Mazoyer B, Joliot M (2002) Automated anatomical labeling of activations in SPM using a macroscopic anatomical parcellation of the MNI MRI single-subject brain. Neuroimage 15:273-289. CrossRef Medline
Weiskopf N, Hutton C, Josephs O, Deichmann R (2006) Optimal EPI parameters for reduction of susceptibility-induced BOLD sensitivity losses: A whole-brain analysis at $3 \mathrm{~T}$ and $1.5 \mathrm{~T}$. Neuroimage 33:493-504. CrossRef Medline

Wiech K, Lin CS, Brodersen KH, Bingel U, Ploner M, Tracey I (2010) Anterior insula integrates information about salience into perceptual decisions about pain. J Neurosci 30:16324-16331. CrossRef Medline

Winston JS, Vlaev I, Seymour B, Chater N, Dolan RJ (2014) Relative valuation of pain in human orbitofrontal cortex. J Neurosci 34:14526-14535. CrossRef Medline

Zacny JP (2010) A possible link between sensation-seeking status and positive subjective effects of oxycodone in healthy volunteers. Pharmacol Biochem Behav 95:113-120. CrossRef Medline

Zuckerman M (1994) Behavioral expressions and biosocial bases of sensation seeking. Cambridge, UK: Cambridge UP. 